\title{
Hyperfine Splitting in Muonic Hydrogen: QED Corrections of the $\alpha^{2}$ Order
}

\author{
S. G. Karshenboim ${ }^{a, b}$, E. Yu. Korzinin ${ }^{a}$, and V. G. Ivanov ${ }^{a, c}$ \\ ${ }^{a}$ State Research Center Mendeleyev All-Russian Institute for Metrology, St. Petersburg, 190005 Russia \\ e-mail: s.g.karshenboim@vniim.ru \\ ${ }^{b}$ Max-Planck-Institut für Quantenoptik, 85748 Garching, Germany \\ ${ }^{c}$ Pulkovo Observatory, Russian Academy of Sciences, Pulkovskoe sh. 65, St. Petersburg, 196140 Russia
}

Received October 8, 2008

\begin{abstract}
The $\alpha^{2}$ order corrections to the hyperfine splitting of the $1 s$ and $2 s$ states in muonic hydrogen have been determined. The specially normalized difference $8 E_{\mathrm{hfs}}(2 s)-E_{\mathrm{hfs}}(1 s)$, as well as the general situation with the theoretical calculations of hyperfine splitting in muonic hydrogen, is considered.
\end{abstract}

PACS numbers: 12.20.Fv, 31.30.Jr, 36.10.Ee

DOI: $10.1134 / \mathrm{S} 0021364008220013$

Hyperfine splitting in a number of simple atomic systems is sensitive to the nuclear structure and its ab initio calculations have a limited accuracy. However, it can be used to compare theoretical results with experimental data, to determine various effective parameters describing the nuclear structure, and to use their values in other calculations.

The investigation of muonic atoms is of considerable interest in view of the determination of the charge radii of various nuclei. In particular, current efforts are focused on measuring the Lamb shift in muonic hydrogen [1]. It is expected that the hyperfine splitting of the $2 s$ metastable state will be measured in this experiment. At the same time, the measurement of the hyperfine splitting of the ground state in a hydrogen atom is designed [2].

One experiment is at a stage far from completion and the other experiment is only designed; thus, the discussion of their accuracy is premature. Nevertheless, it is desired to calculate all corrections comparable with $100 \mathrm{ppm}$ and to have a strategy for using the experimental results if they are more accurate than this value.

For the former problem, we calculate the single unknown electrodynamic correction of interest. It contributes about $59 \mathrm{ppm}$ to the hyperfine splitting of the $1 \mathrm{~s}$ state. The other unknown quantum electrodynamic (QED) corrections are much smaller.

At the same time, the error in the calculation of a number of corrections associated with the nuclear structure can exceed $100 \mathrm{ppm}$. To overcome this difficulty, a solution proposed for light hydrogen-like atoms $[3,4]$ can be used; this solution is the calculation of the difference

$$
\Delta E_{21}=8 E_{\mathrm{hfs}}(2 s)-E_{\mathrm{hfs}}(1 s) .
$$

The feature of this difference is the cancellation of the "hard" contributions to the energy of the $n l$ states, which are usually proportional to the square of the wavefunction at the origin,

$$
\left|\Psi_{n l}(\mathbf{r}=0)\right|^{2}=\frac{(Z \alpha)^{3} m_{r}^{3}}{\pi n^{3}} \delta_{0 l} .
$$

Here, $Z$ is the charge number of the nucleus, $\alpha$ is the fine structure constant, $m_{r}$ is the reduced mass of a bound particle (the electron in ordinary atoms and the muon in muonic atoms), and the relativistic system of units is used with $\hbar=c=1$.

In particular, the leading nonrelativistic contribution to the hyperfine splitting in hydrogen-like atoms is determined by the $\delta$-function potential and does not contribute to difference (1):

$$
E_{\mathrm{hfs}}^{(0)}(n s)=\frac{E_{\mathrm{F}}}{n^{3}},
$$

where

$$
E_{\mathrm{F}}=\frac{8}{3} \alpha(Z \alpha)^{3} m \frac{m}{m_{p}} \frac{\mu}{\mu_{N}}\left(\frac{m_{r}}{m}\right)^{3}=182443 \times 10^{-6} \mathrm{eV}
$$

is the so-called Fermi energy. ${ }^{1}$ Here, $\mu_{N}$ is the nuclear magneton, $\mu$ is the magnetic moment of the nucleus $(I=$ $1 / 2$ is assumed for spin), $m$ is the mass of the bound particle (electron or muon), and $m_{p}$ is the proton mass.

The uncertainty in theoretical calculations is associated with the nuclear structure effects. The leading nuclear structure contributions are determined by twophoton exchanges with a high momentum transfer and

\footnotetext{
${ }^{1}$ The numerical values are given for muonic hydrogen.
} 
have the hard structure and, therefore, cancel when calculating difference (1).

The ab initio calculations of this difference in light hydrogen-like atoms ensure a much higher absolute accuracy than calculations of the hyperfine splitting separately for the states in this difference. The result is sensitive to the QED corrections of the third and fourth orders in the parameters $\alpha, Z \alpha$, and mass ratio $m / M[5$, $6]$. The theoretical calculations can be critically compared with the existing results of measuring the hyperfine splitting of $1 s$ and $2 s$ levels in hydrogen [7,6], deuterium [8, 6], and helium-3 ion [9]. At the same time, the mentioned corrections are not of current importance for comparison with the existing or expected experimental data on the hyperfine splitting of the $1 s$ and $2 s$ levels separately, because they are much smaller than the errors in the nuclear-structure corrections. A similar calculation can be performed for muonic hydrogen in view of the expected experimental results $[2,1]$ (see also [10]). Calculations [10] performed several years ago included only corrections of the first order in $\alpha$ and the leading theoretical error was associated with twoloop polarization contributions. In this work, we calculate these contributions for the $1 s$ and $2 s$ states and analyze the higher order corrections associated with the nuclear structure. In conclusion, we discuss the accuracy of calculating difference (1) in muonic hydrogen and prospects for the comparison of theoretical results with experimental data.

A large number of the known corrections were derived for ordinary, rather than muonic, atoms [3, 4]. Here, we briefly discuss them in application to muonic hydrogen. The leading QED contributions to the hyperfine splitting are given by the expression

$$
E_{\mathrm{hfs}}^{e \mathrm{QED}}(n s)=E_{\mathrm{hfs}}^{(0)}(n s)\left[1+Q_{\mathrm{QED}}^{e}(n s)\right] .
$$

Here (see, e.g., [11]),

$$
\begin{gathered}
Q_{\mathrm{QED}}^{e}(1 s)=a+\left\{\frac{3}{2}(Z \alpha)^{2}+\alpha(Z \alpha)\left(\ln 2-\frac{5}{2}\right)\right. \\
+\frac{\alpha(Z \alpha)^{2}}{\pi}\left[-\frac{2}{3} \ln \frac{1}{(Z \alpha)^{2}}\left(\ln \frac{1}{(Z \alpha)^{2}}\right.\right. \\
\left.\left.+4 \ln 2-\frac{281}{240}\right)+16.903772 \ldots\right] \\
\left.+0.7718(4) \frac{\alpha^{2}(Z \alpha)}{\pi}\right\},
\end{gathered}
$$

where $a$ is the anomalous moment of the bound particle (the electron in hydrogen or the muon in muonic hydrogen). The expression contains the electrodynamic corrections identical for muonic and ordinary atoms and does not include the effects of a finite size and structure of the nucleus. Among the latter corrections are the recoil corrections, which imply integration over high momentum transfers when the nuclear form factors and other more complex effects should be taken into account [12].

For the normalized difference given by Eq. (1), the situation with the recoil corrections is significantly different, because the "hard" loop integrations requiring the inclusion of the nuclear-structure effects lead to $\delta$-function potentials and do not contribute to Eq. (1). The QED part of the difference can include additional terms. In particular, the corrections of the order $(Z \alpha)^{2}(m / M)$ in the general case (e.g., for the $1 s$ state) are not pure electrodynamic corrections as for difference (1) for which the QED result has the form [3, 5, 13]

$$
\begin{gathered}
\Delta E_{21}^{(e \mathrm{QED})}=(Z \alpha)^{2} E_{\mathrm{F}}\left\{\frac{5}{8}\right. \\
+\frac{\alpha}{\pi}\left[\left(7-\frac{16}{3} \ln 2\right) \ln \frac{1}{Z \alpha}-5.55155 \ldots\right] \\
+\frac{m}{M}\left[-\frac{9}{8}+\left(\frac{\ln 2}{2}-\frac{7}{32}\right)\left(1-\frac{1}{\eta}\right)\right. \\
\left.\left.-\left(\frac{145}{128}-\frac{7}{8} \ln 2\right) \eta\right]\right\}=1.98 \times 10^{-6} \mathrm{eV},
\end{gathered}
$$

where $M$ is the nuclear mass and the parameter

$$
\eta=\frac{\mu}{\mu_{N} I} \frac{M}{m_{p}} \frac{1}{Z}
$$

has the meaning of $g$ factor.

The corrections marked by the superscript $e \mathrm{QED}$ are identical for electron and muonic atoms, but the uncertainty of their calculations are significantly different. In both cases, uncertainty is associated with the contributions of the fourth order $\delta E_{21}^{(e \mathrm{QED})}$, which additionally include one of the parameters $\alpha, Z \alpha$, or $m / M$. These corrections are known only partially [5, 6, 14]. The corrections with additional factors $\alpha$ and $Z \alpha$ dominate for electron atoms. Only corrections associated with the recoil effects are significant for muonic hydrogen [recall that $(m / M)_{e} \simeq 1 / 1836 \ll \alpha=Z \alpha \simeq 1 / 137 \ll$ $(m / M)_{\mu} \simeq 1 / 9$ for hydrogen and muonic hydrogen]. Some of them are known in the logarithmic approximation [5]:

$$
\begin{gathered}
\delta E_{21}^{(e \mathrm{QED})}=(Z \alpha)^{2} E_{\mathrm{F}}\left\{\frac{\alpha}{\pi} \frac{m}{M}\left(\frac{32}{3} \ln 2-14\right)\right. \\
\left.+\frac{Z \alpha}{\pi} \frac{m}{M}\left(2-\frac{4}{3} \ln 2\right)\right\} \ln \frac{1}{Z \alpha} .
\end{gathered}
$$

The uncertainty is estimated as the half of the logarithmic contribution: $\delta E_{21}^{(e Q E D)}=-0.069(34) \times 10^{-6} \mathrm{eV}$. In 
the uncertainty for muonic hydrogen, the $(Z \alpha)^{2}(m / M)^{2}$ contribution dominates; it is unknown because it is disregarded in the problem for usual atoms [5].

The leading contribution to the difference $\Delta E_{21}$ in ordinary atoms is of the order $(Z \alpha)^{2} E_{\mathrm{F}}$; however, for muonic atoms, there is an additional contribution of the first order in $\alpha$ (see Table 1) [10], which dominates and changes the contribution of the difference. Such specific muon QED contributions are given by the expressions

$$
\begin{gathered}
\Delta E^{(\mu \mathrm{QED})}(n s)=\frac{\alpha}{\pi}\left(c_{1}(n)+\frac{\alpha}{\pi} c_{2}(n)\right)\left(1+a_{\mu}\right) E_{\mathrm{hfs}}^{(0)}(n s), \\
\Delta E_{21}^{(\mu \mathrm{QED})}=\frac{\alpha}{\pi}\left(c_{1}(21)+\frac{\alpha}{\pi} c_{2}(21)\right)\left(1+a_{\mu}\right) E_{\mathrm{F}} .
\end{gathered}
$$

Until recently [10], the uncertainty in the calculations of the electrodynamic part $\Delta E_{21}$ was determined by the unknown two-loop contribution to $\Delta E_{21}^{(\mu \mathrm{QED})}$. The calculation of the coefficient $c_{2}$ is the main result of this work.

The specific higher order QED contributions include both unknown three-loop vacuum-polarization contributions of the order $(\alpha / \pi)^{3} E_{\mathrm{F}}$ and relativistic corrections to the single-loop contribution of the order $(\alpha / \pi)(Z \alpha)^{2} E_{\mathrm{F}}$. The latter corrections are known for the states $1 s[15], 2 s[5]$, and $\delta E_{21}^{(\mu \mathrm{QED})}=1.5 \frac{\alpha}{\pi}(Z \alpha)^{2} E_{\mathrm{F}}=$ $0.033 \times 10^{-6} \mathrm{eV}$.

In this work, we calculate the contributions of the second order in the vacuum polarization. To this end, it is necessary to consider the contributions of the first, second, and third orders of nonrelativistic perturbation theory. The corresponding diagrams and results for individual contributions are summarized in Table 2 . The second-order polarization contribution is $-1.72842 \times$ $10^{-6} \mathrm{eV}$ and the total result for specific muon contributions is $\Delta E_{21}^{(\mu \mathrm{QQD})}=-128.878(2) \times 10^{-6} \mathrm{eV}$.

We briefly describe the basic elements of the calculation. The key blocks in the diagrams presented in Table 2 include the electrostatic and magnetic interactions induced by vacuum polarization and nonrelativistic Green's function for muon in the Coulomb field. We represent the vacuum polarization by means of the dispersion integral so that the corresponding electrostatic potential has the form

$$
-\frac{Z \alpha}{r} \int_{0}^{1} d v \rho(v) \exp \left[-\frac{2 m_{e} r}{\sqrt{1-v^{2}}}\right],
$$

use the known spectral functions $\rho$ for the irreducible Uehling and Källen-Sabry potentials (see, e.g., [16]),
Table 1. Contributions of the order $\alpha E_{\mathrm{F}}$ to the hyperfine splitting in muonic hydrogen [10]. Contribution (a) corresponds to the correction for electron vacuum polarization in a transverse photon and contribution (b) presents the effect of the electrostatic Uehling potential on the hyperfine splitting.

\begin{tabular}{|c|c|c|c|c|}
\hline \multicolumn{2}{|r|}{ Contribution } & $c_{2}(1 s)$ & $c_{2}(2 s)$ & $c_{2}(21)$ \\
\hline $\mathrm{a}$ & & 0.883041 & 0.91026 & 0.027219 \\
\hline $\mathrm{b}$ & 2 & 1.73115 & 1.40425 & -0.326906 \\
\hline & Total & 2.61419 & 2.31451 & -0.299687 \\
\hline
\end{tabular}
The double line corresponds to the Coulomb Green's function of the muon

Table 2. Contributions of the electron vacuum polarization of the second order to the hyperfine splitting in muonic

\begin{tabular}{|c|c|c|c|c|}
\hline \multicolumn{2}{|r|}{ Contribution } & $c_{2}(1 s)$ & $c_{2}(2 s)$ & $c_{2}(21)$ \\
\hline $\mathrm{a}$ & & 1.28028 & 1.30453 & 0.02425 \\
\hline $\mathrm{b}$ & & 1.68287 & 1.73944 & 0.05657 \\
\hline $\mathrm{c}$ & 2 & 1.85512 & 1.73785 & -0.11727 \\
\hline d & 2 & 3.79665 & 2.83394 & -0.96271 \\
\hline $\mathrm{e}$ & 2 & 1.16347 & 1.02936 & -0.13410 \\
\hline $\mathrm{f}$ & & 0.92294 & 0.56352 & -0.35942 \\
\hline $\mathrm{g}$ & & 0.68104 & 0.41990 & -0.26114 \\
\hline & Total & 11.3824 & 9.62854 & -1.75382 \\
\hline
\end{tabular}
hydrogen. Contributions (b) and (d) correspond to the total irreducible part of the two-loop polarization operator 
and represent the reducible two-loop polarization in the form

$$
\begin{gathered}
\rho_{1 \cdot 1}(v)=-\frac{1}{9}\left(\frac{\alpha}{\pi}\right)^{2} \frac{v^{2}\left(1-v^{2} / 3\right)}{1-v^{2}} \\
\times\left\{16-6 v^{2}+3 v\left(3-v^{2}\right) \ln \left(\frac{1-v}{1+v}\right)\right\} .
\end{gathered}
$$

The corresponding magnetic interaction is expressed in terms of the derivatives of the electrostatic potential. The unified representation of the potentials simplifies the calculation of radial integrals (see below) and makes it possible to perform a number of tests on the initial formulas and programs for their calculation (by comparing the calculated contributions in Table 2 with known contributions in Table 1).

At the same time, the nonrelativistic Coulomb Green's function, which is another important element of the diagrams, is represented in the form of the expansion in the solutions of the Sturm-Liouville problem [17]:

$$
\begin{aligned}
& G\left(E ; \mathbf{r}^{\prime}, \mathbf{r}\right)=\frac{1}{Z \alpha} \sum_{l m_{l}} \sum_{k=l+1}^{\infty} \frac{v}{k-v} \\
& \times \Phi_{k l}^{(v)}(r) \Phi_{k l}^{(v)}\left(r^{\prime}\right) Y_{l m_{l}}^{*}(\mathbf{\Omega}) Y_{l m_{l}}\left(\mathbf{\Omega}^{\prime}\right) .
\end{aligned}
$$

The solutions of the Sturm-Liouville problem $\Phi_{n l m_{l}}^{(\mathrm{v})}(\mathbf{r})=n(Z \alpha m)^{-1 / 2} \Psi_{n l m_{l}}\left(\frac{n}{v} \mathbf{r}\right)$ with the energy $E=$ $-(Z \alpha)^{2} m / 2 v^{2}$ have a form similar to the wavefunctions of the bound states in the hydrogen atom $\Psi_{n l m_{l}}(\mathbf{r})$. This representation is in many aspects similar to the usual sum over the states in the Coulomb problem, but only the discrete spectrum is present. The matrix elements of the interactions are calculated in the coordinate representation and are completely similar to those analyzed in our previous works [18-20].

Several simplifications reduce the number of sums and integrals in the contributions of a number of diagrams. In particular, the application of the dispersion representation for the reducible two-loop polarization in the form $\rho_{1 \cdot 1}(v)$ reduces the number of integrations with respect to the dispersion parameters. ${ }^{2}$

\footnotetext{
2 The reducible part of vacuum polarization is usually represented using a double integral. In this case, a correction to the photon propagator in the momentum representation vanishes at zero momentum transfer. In this representation, the correction is proportional to $\int d v \rho_{1 \cdot 1}(v)\left[q^{2}-4 m_{e}^{2} /\left(1-v^{2}\right)\right]^{-1}$ and vanishes at $q^{2}=0$, because $\int d v \rho_{1 \cdot 1}(v)\left(1-v^{2}\right)=0$.
}

Several contributions (see Table 2, contributions $c$, $\mathrm{d}$, and $\mathrm{f}$ ) involve the characteristic convolution

$$
\delta \Psi_{n s}^{\mathrm{hfs}}(\mathbf{r})=\int d^{3} \mathbf{r}^{\prime} \Psi_{n s}\left(\mathbf{r}^{\prime}\right) V_{\mathrm{hfs}}\left(\mathbf{r}^{\prime}\right) \tilde{G}_{n s}\left(E_{n} ; \mathbf{r}^{\prime}, \mathbf{r}\right)
$$

which can be reduced to the expression

$$
\delta \Psi_{n s}^{\mathrm{hfs}}(\mathbf{r})=\frac{E_{\mathrm{hfs}}^{(0)}(n s)}{\Psi_{n s}(\mathbf{r}=0)} \tilde{G}_{n s}\left(E_{n} ; 0, \mathbf{r}\right) .
$$

Here, the reduced Green's functions $\tilde{G}$ are given by the explicit formulas, e.g., [21]:

$$
\tilde{G}_{1 s}\left(E_{1} ; 0, \mathbf{r}\right)=\frac{Z \alpha m^{2}}{\pi} e^{-\frac{z}{2}}\left(\ln z+C+\frac{z^{2}-5 z-2}{2 z}\right),
$$

where $C$ is the Euler constant and $z=2 Z \alpha m r$. As a result, one summation over the intermediate states and one integration with respect to the radius are trivial and the integration with respect to the radius that involves $\delta \Psi_{n s}^{\mathrm{hfs}}(\mathbf{r})$ is similar to those considered in [15, 22].

The general expression in the third order of perturbation theory in the potential $\delta V$ (see the last two contributions in Table 2),

$$
\begin{aligned}
\Delta E_{\mathrm{hfs}}^{(3)}(n s)= & \left\langle\Psi_{n s}(\mathbf{r})\right| \delta V(\mathbf{r}) \tilde{G}\left(\mathbf{r}, \mathbf{r}^{\prime}\right)\left[\delta V\left(\mathbf{r}^{\prime}\right)-\delta E_{n s}^{(1)}\right] \\
& \times \tilde{G}\left(\mathbf{r}^{\prime}, \mathbf{r}^{\prime \prime}\right) \delta V\left(\mathbf{r}^{\prime \prime}\right)\left|\Psi_{n s}\left(\mathbf{r}^{\prime \prime}\right)\right\rangle,
\end{aligned}
$$

where $\delta E_{n s}^{(1)}=\left\langle\Psi_{n s}|\delta V| \Psi_{n s}\right\rangle$, includes the subtraction terms, which can be simplified because

$$
\int d^{3} \mathbf{r} \Phi_{n s}^{(n)}(\mathbf{r})^{*} \Phi_{n^{\prime} s}^{(n)}(\mathbf{r})=0 \text {, if }\left|n-n^{\prime}\right| \geq 2 .
$$

In view of the mentioned simplifications, the most complex contributions include only double integrals with respect to the radius and a single sum over intermediate states; all integrals with respect to the radius are calculated analytically (cf. $[18,15])$ and the integrals with respect to the dispersion variable $v$ are calculated both analytically and numerically (cf. [18-20, 22]).

The calculation of the nuclear-structure corrections is a nuclear problem rather than an atomic problem. However, when calculating the corrections to normalized difference (1), we can use phenomenological $\delta$-function potentials [5], which, in particular, successfully describe the contributions in the leading order. Two such contributions are proportional to the $\delta$ function and lead to the correction $\Delta E_{\mathrm{hfs}}^{\mathrm{Nucl}}(1 s)$ to the hyperfine splitting, and to the Lamb shift (the potential proportional to the square of the nuclear charge radius $R_{E}$ ), respectively. There is one contact term that has the form of the Laplacian of the $\delta$ function and is proportional to 
the square of the magnetic radius $R_{M}$. The contribution from the nuclear structure effects has the form $[5,6,23]$

$$
\begin{gathered}
\Delta E_{21}^{e \text { Nucl }}=(Z \alpha)^{2}\left\{\left(\ln 2+\frac{3}{16}\right) \Delta E_{\mathrm{hfs}}^{e \text { Nucl }}(1 s)\right. \\
\left.+\left(m_{r} R_{E}\right)^{2} E_{\mathrm{F}}\left[2-\frac{4}{3} \ln 2-\frac{1}{4}\left(\frac{R_{M}}{R_{E}}\right)^{2}\right]\right\},
\end{gathered}
$$

where the charge and magnetic radii of the proton are defined as the rms radii. These contributions are of approximately the same order in usual hydrogen (because $m_{r} R_{p} \simeq 0.002$ ). For muonic hydrogen, where $m_{r} R_{p} \simeq 0.4$, the contributions proportional to $\left(m R_{p}\right)^{2}$ are of the order $(Z \alpha)^{2} E_{\mathrm{F}}$ and the first contribution is of the order $(Z \alpha)^{3} E_{\mathrm{F}}$ because $\Delta E_{\mathrm{hfs}}^{\mathrm{Nucl}}(1 s) \sim(Z \alpha)\left(m_{r} R_{p}\right) E_{\mathrm{F}}$.

As mentioned in [10], there is also a specific muon contribution

$$
\Delta E_{21}^{\mu \text { Nucl }}=\frac{\alpha}{\pi} c_{1}^{b} \Delta E_{\mathrm{hfs}}^{e \text { Nucl }}(1 s) .
$$

Here, it is taken into account that the value of the nonrelativistic wavefunction at the origin is modified by the Uehling potential.

The sum of the above contributions is given by the expression

$$
\begin{gathered}
\Delta E_{21}=\left(-126.93(49)+1.03(1) \frac{\Delta E_{\mathrm{hfs}}^{e \text { Nucl }}(1 s)}{\Delta E_{\mathrm{hfs}}^{e \text { Nucl, } 0}(1 s)}\right. \\
\left.+1.96(2)\left(\frac{R_{E}}{R_{0}}\right)^{2}-0.46(1)\left(\frac{R_{M}}{R_{0}}\right)^{2}\right) \times 10^{-6} \mathrm{eV},
\end{gathered}
$$

which includes three parameters $\Delta E_{\mathrm{hfs}}^{\mathrm{Nucl}}(1 s), R_{E}$, and $R_{M}$. To estimate the orders of magnitudes, we use rough estimates for the radii and nuclear-structure corrections. Here, $R_{0}=0.9 \mathrm{fm}$, and we used a rough estimate $\left(\Delta E_{\mathrm{hfs}}^{e \mathrm{Nucl}, 0}(1 s)=-1450.72 \times 10^{-6} \mathrm{eV}\right)$ for the normalization of the leading correction $\Delta E_{\mathrm{hfs}}^{\mathrm{Nucl}}(1 s)$. The deviation of the real values of the parameters $\Delta E_{\mathrm{hfs}}^{e \text { Nucl }}(1 s), R_{E}$, and $R_{M}$ are noticeably smaller than unity and such parameterization is very convenient for estimating the uncertainties of various contributions.

Let us discuss the accuracy of the resulting expression. The uncertainty of the specific muon contributions $\Delta E_{21}^{\mu \mathrm{QED}}$ is determined by the unknown thirdorder contributions $(\alpha / \pi)^{3} E_{\mathrm{F}}$. An uncertainty associated with the contribution $(\alpha / \pi)^{2} E_{\mathrm{F}}$ calculated in this work dominated previously in the theoretical expression [10].
The uncertainty of the standard QED theory common for the electron and muon, $\Delta E_{21}^{e \mathrm{QED}}$, is associated with the unknown contributions of the order $(Z \alpha)^{2}(m / M)^{2} E_{\mathrm{F}}$. This contribution determines the uncertainty of the QED calculations at present. Similar calculations have already been performed $[4,24]$ and it should be expected that this contribution will be calculated soon. After that, the uncertainty of the electrodynamic contributions will be determined by other higher order corrections $\frac{\alpha}{\pi}(Z \alpha)^{2} \frac{m}{M} E_{\mathrm{F}}$ and $(Z \alpha)^{3} \frac{m}{M} E_{\mathrm{F}}$, which are known [5] in the logarithmic approximation specified by Eq. (7).

When analyzing the nuclear structure contributions, it is necessary to separate the accuracy with which the coefficients in Eq. (14) are determined and the accuracy with which the nuclear parameters appearing in them are known. The nonrelativistic correction $\Delta E_{21}^{\mu \text { Nucl }}$ is known except for the higher order nonrelativistic effects; the inclusion of the relativistic effects leads to the additional factor $(Z \alpha)^{2}$. The coefficient is calculated with a percent accuracy.

The contributions to $\Delta E_{21}^{e \text { Nucl }}$ contain two types of terms. The terms explicitly including the nuclear radius are of nonrelativistic origin and the corrections to them are at the percent level, whereas a small relativistic correction containing $\Delta E_{\mathrm{hfs}}^{e \text { Nucl }}(1 s)$ is associated with the relativistic calculations and can have the relative order $m / M$.

Thus, the uncertainty is currently determined by an unknown contribution of the order $(Z \alpha)^{2}(m / M)^{2} E_{\mathrm{F}}$ and, after its calculation, will be determined by an accuracy with which the parameters entering into the expression are known.

The parameters characterizing the nuclear structure can be considered in different ways. The first way is based on the existing values of the radii $R_{E}$ and $R_{M}$, which are determined primarily from the scattering data [25]; the charge radius is measured with a higher accuracy than the magnetic radius. In addition, the electric charge radius of the proton is extracted from the spectroscopy of the hydrogen atom [26]. The nuclear structure correction $\Delta E_{\mathrm{hfs}}^{\mathrm{Nucl}}(1 s)$ can be estimated from relatively realistic models.

We prefer another strategy. Recall that the calculation of the difference $\Delta E_{21}$ is of applied meaning only in the case of the success in the experiments on the measurement of the hyperfine splitting in muonic hydrogen $[1,2]$. The accuracy of these experiments will directly determine the accuracy of the experimental determination of the difference $\Delta E_{21}$. Note that it is easy to determine $\Delta E_{\mathrm{hfs}}^{\mathrm{Nucl}}(1 s)$, because it can be extracted by directly comparing the QED theory discussed above 
with the measurement of the hyperfine splitting of the $1 s$ state in muonic hydrogen:

$$
\Delta E_{\mathrm{hfs}}^{e \text { Nucl }}(1 s)=\frac{E_{\mathrm{hfs}}^{\mathrm{exp}}(1 s)-E_{\mathrm{hfs}}^{\mathrm{QED}}(1 s)}{1+c_{1}^{b}(1 s) \frac{\alpha}{\pi}} .
$$

The appearing contribution multiplied by $\alpha / \pi$ has the uncertainty associated with the parameter $\Delta E_{\mathrm{hfs}}^{\mathrm{Nucl}}(1 s)$ negligible as compared to the uncertainty in the experimental $\Delta E_{21}$ value.

The accuracy of determining the proton radii is satisfactory for comparing theoretical results and experimental data for difference (1) (at realistic expectations of the accuracies of the experiments [1,2]). This comparison will make it possible to verify the consistency of the data of two experiments with muonic hydrogen. This will be very useful in view of the complexity of the experiments $[1,2]$. If the uncertainty in determining the radii is dominating in a certain scenario after an increase in the experimental accuracy, the comparison of the theoretical results and experimental data for difference (1) will allow a more accurate determination of this parameter.

We are grateful to V.A. Shelyuto for stimulating discussions. This work was supported by the Russian Foundation for Basic Research (project no. 08-0291969) and by Deutsche Forschungsgemeinschaft (grant no. GZ 436 RUS 113/769/0-3). The work of E.Yu.K. was also supported by the Dynasty Foundation.

\section{REFERENCES}

1. T. Nebel et al., Can. J. Phys. 85, 469 (2007).

2. P. Regoliosi, "QCL-MUH: Tunable Sources for Measurements of Proton Polarizability," Presented at Opening Meeting of Hadron Physics 2, LNF, Frascati, Italy, Sept. 28-29, 2007.

3. D. Zwanziger, Phys. Rev. 121, 1128 (1961).

4. M. Sternheim, Phys. Rev. 130, 211 (1963).

5. S. G. Karshenboim and V. G. Ivanov, Phys. Lett. B 524, 259 (2002); Euro. Phys. J. D 19, 13 (2002); S. G. Karshenboim, in The Hydrogen Atom: Precision Physics of Simple Atomic Systems, Ed. by S. G. Karshenboim et al. (Springer, Berlin, 2001), p. 335.

6. S. G. Karshenboim, N. N. Kolachevsky, V. G. Ivanov, et al., Zh. Éksp. Teor. Fiz. 129, 419 (2006) [JETP 102, 367 (2006)].

7. N. Kolachevsky, M. Fischer, S. G. Karshenboim, and T. W. Hänsch, Phys. Rev. Lett. 92, 033003 (2004).
8. N. Kolachevsky, P. Fendel, S. G. Karshenboim, and T. W. Hänsch, Phys. Rev. A 70, 050412 (2004).

9. M. H. Prior and E. C. Wang, Phys. Rev. A 16, 6 (1977).

10. K. Jungmann, V. G. Ivanov, and S. G. Karshenboim, in The Hydrogen Atom: Precision Physics of Simple Atomic Systems, Ed. by S. G. Karshenboim et al. (Springer, Berlin, 2001), p. 446.

11. M. I. Eides, H. Grotch and V. A. Shelyuto, Phys. Rept. 342, 63 (2001); Theory of Light Hydrogenic Bound States, Springer Tracts Mod. Phys. (Springer, Berlin, 2007), Vol. 222.

12. G. T. Bodwin, D. R. Yennie, and M. Gregorio, Phys. Rev. Lett. 48, 1799 (1982); Rev. Mod. Phys. 57, 723 (1985).

13. S. G. Karshenboim, Phys. Rep. 422, 1 (2005).

14. S. G. Karshenboim and V. G. Ivanov, Can. J. Phys. 83, 1063 (2005); V. A. Yerokhin, A. N. Artemyev, V. M. Shabaev, and G. Plunien, Phys. Rev. A 72, 052510 (2005).

15. S. G. Karshenboim, V. G. Ivanov, and V. M. Shabaev, Zh. Éksp. Teor. Fiz. 117, 67 (2000) [JETP 90, 59 (2000)]; S. G. Karshenboim, V. G. Ivanov, and V. M. Shabaev, Can. J. Phys. 76, 503 (1998).

16. M. I. Eides, S. G. Karshenboim, and V. A. Shelyuto, Phys. Lett. B 229, 285 (1989); Yu. Schwinger, Particles, Sources, and Fields, Vol. 2 (Westview, Boulder, Colorado, 1998).

17. P. P. Pavinsky and A. I. Sherstyuk, Vestnik Len. Gos. Univ. 22, 11 (1968); L. Hostler, J. Math. Phys. 11, 2966 (1970); S. V. Khristenko and S. I. Vechnikin, Opt. Spektrosk. 31, 503 (1971); S. A. Zapryagaev, N. L. Manakov, and L. P. Rapoport, Yad. Fiz. 15, 508 (1972) [Sov. J. Nucl. Phys. 15, 282 (1972)].

18. S. G. Karshenboim, Zh. Éksp. Teor. Fiz. 116, 1575 (1999) [JETP 89, 850 (1999)]; S. G. Karshenboim, Can. J. Phys. 76, 169 (1998).

19. S. G. Karshenboim, V. G. Ivanov, and E. Yu. Korzinin, Eur. Phys. J. D 39, 351 (2006); E. Yu. Korzinin, V. G. Ivanov, and S. G. Karshenboim, Eur. Phys. J. D 41, 1 (2007).

20. E. Yu. Korzinin, V. G. Ivanov, and S. G. Karshenboim, arXiv:0712.2114.

21. V. G. Ivanov and S. G. Karshenboim, Zh. Éksp. Teor. Fiz. 109, 1219 (1996) [JETP 82, 656 (1996)].

22. S. G. Karshenboim, U. Jentschura, V. Ivanov, and G. Soff, Eur. Phys. J. D 2, 209 (1998).

23. S. G. Karshenboim, Phys. Lett. A 225, 97 (1997).

24. K. Pachucki, Phys. Rev. Lett. 79, 4120 (1997); Phys. Rev. A 56, 297 (1997); K. Pachucki and S. G. Karshenboim, Phys. Rev. Lett. 80, 2101 (1998).

25. I. Sick, Prog. Part. Nucl. Phys. 47, 245 (2001).

26. P. J. Mohr, B. N. Taylor, and D. B. Newell, Rev. Mod. Phys. 80, 633 (2008).

Translated by R. Tyapaev 